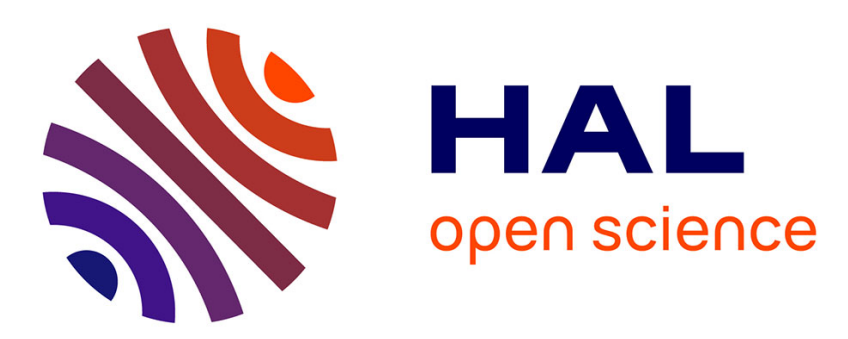

\title{
Interference-aware multipath routing protocol for video transmission over ZigBee wireless sensor networks
}

\author{
Zahia Bidai, Moufida Maimour
}

\section{To cite this version:}

Zahia Bidai, Moufida Maimour. Interference-aware multipath routing protocol for video transmission over ZigBee wireless sensor networks. 4th International Conference on Multimedia Computing and Systems, ICMCS14, IEEE, Apr 2014, Marrakesh, Morocco. pp.837-842, 10.1109/ICMCS.2014.6911267 . hal-01087934

\section{HAL Id: hal-01087934 \\ https://hal.science/hal-01087934}

Submitted on 27 Nov 2014

HAL is a multi-disciplinary open access archive for the deposit and dissemination of scientific research documents, whether they are published or not. The documents may come from teaching and research institutions in France or abroad, or from public or private research centers.
L'archive ouverte pluridisciplinaire HAL, est destinée au dépôt et à la diffusion de documents scientifiques de niveau recherche, publiés ou non, émanant des établissements d'enseignement et de recherche français ou étrangers, des laboratoires publics ou privés. 


\section{Interference-Aware Multipath Routing Protocol for Video Transmission over ZigBee Wireless Sensor Networks}

\author{
Zahia Bidai* \\ Laboratory of Research in Industrial \\ Computing and Networking, LRIIR \\ Oran University, BP 1524 El M'naouar, Algeria \\ bidai.zahia@univ-oran.dz
}

\author{
Moufida Maimour ${ }^{\dagger}$ \\ † Lorraine University, CRAN, UMR 7039 \\ Campus Sciences, BP 70239 \\ Vandœuvre-lès-Nancy Cedex, 54506, France \\ ${ }^{*}$ CNRS, CRAN, UMR 7039, France \\ moufida.maimour@univ-lorraine.fr
}

\begin{abstract}
The IEEE 802.15.4/ZigBee technology is emerging as an important wireless sensor networking solution for short and medium range communication due to its numerous advantages. On the other hand, multimedia applications have gained increasing popularity that gives us the opportunity to improve this technology to support video transmission. In limited bandwidth ZigBee networks $(250 \mathrm{kbps}$ at $2.4 \mathbf{G H z}$ ), how to increase the throughput in constrained environments has become the main challenge. Multi-path becomes a crucial feature in supporting video transmission. Using simultaneous multiple paths provides the bandwidth requirements of multimedia applications. Unfortunately, concurrent transmission creates the problem of inter-path interference mainly due to the broadcast nature of the underlying medium. In this paper, we propose an interference-minimized multipath routing to facilitate MPEG-4 video transmission over ZigBee-based WSNs. This latter is evaluated using comparison with an interfering multipath routing version and the single path routing under different video frame rates. Results show that the proposed interference-aware multipath routing for ZigBee-based WSNs can dramatically reduce the level of interference and thus maximize the overall throughput and improve the received video quality.
\end{abstract}

Index Terms-IEEE 802.15.4/ZigBee, MPEG-4 video, Multipath routing, Interference, Performance evaluation.

\section{INTRODUCTION}

Wireless Multimedia Sensor Networks (WMSNs) have been developed with the availability of low-cost cameras and microphones which allow a tiny sensor to be equipped with modules for collecting visual and audio informations. WMSN can significantly enhance a wide range of applications like object detection, recognition, tracking and video surveillance, etc. However, multimedia applications produce a huge amount of data to be transmitted with high reporting rates.

The importance of WSN has been enforced by the delivery of the most popular ZigBee technology [1]. ZigBee is a standard for low rate wireless personal area networks (LR-WPANs) which is designed for low cost, low power and short range communications making it very suitable for WSN. ZigBee networks implement the IEEE 802.15.4 [2] as their physical and MAC layers. However, this technology suffers from its limited bandwidth $(250 \mathrm{kbps}$ at $2.4 \mathrm{GHz})$ and extending it to meet multimedia applications requirements is a real challenge.

Recently, video transmission over low data-rate networks becomes feasible thanks to a number of reasons such as advanced video compression techniques as MPEG4 and H.264 [3], advanced content based video segmentation and rate control algorithms. Such efforts have indeed contributed to reduce the complexity and difficulty of video transmission over such low rate networks. Various works were proposed to handle multimedia and high data applications over ZigBee networks and have focused especially on the MAC/physical layers [4][5].

At the network level, multipath routing approach can be used to satisfy the bandwidth requirement of multimedia applications. This approach, has been one of the most important current direction in the area of routing. It is known for its benefits to Adhoc and WSNs and allows the establishment of multiple paths between one source and a destination node. According to the application goal, multipath routing may be proposed in order to increase the reliability of data transmission (i.e. fault tolerance) or to provide load balancing and higher aggregated bandwidth since data packets can be propagated simultaneously over several paths. However, because a wireless channel is a broadcast medium, transmitting data concurrently over multiples paths seems to be less profitable than transmitting data over a single path and can annul all theoretical benefits of multipath routing if the effects of interpath interference are not controlled.

The overlapping of radio ranges of (some) nodes belonging to paths that are close to each other is known in the literature as the route coupling problem. Generally, the paths that have nodes or links in common are considered as strongly coupled paths. However, the route coupling problem can occur in multi-hop wireless networks even if two paths have no links or nodes in common (node disjoint paths). To reduce the effects of the route coupling problem, geographic routing based on localization is one of the most obvious techniques allowing construction of non-interfering paths [6]. Alternatively, other techniques such as radio resources management 
(using directional transmission [7], multi-channel transmission [8], etc.), scheduling transmission (S-TDMA) [9]) and the use of conflict graphs approach [10] have been proposed to minimize the effects of the inter-path interference. However, geographic routing requires additional localization algorithms which consumes resources. The use of directional antenna to reduce interference also requires specific hardware equipment that may not be attractive for low cost wireless sensor nodes. Furthermore, although the multi-channel communication may increase network throughput and reduce interference, it requires a special mechanism at the MAC layer to support channels switching. This mechanism is costly in terms of energy consumption for WSNs and it is quite suitable for mesh networks than WSNs. Consequently, these approaches may not be effective solutions to reduce the negative effects of interference in many WSN applications with limited resources.

$\mathrm{Wu}$ and Harms [11] introduced a metric called correlation factor, to measure the relative independance degree between two node-disjoint paths. The correlation factor of two paths is defined as the number of links which are inside the transmission range of each other. Another metric was proposed in [12] called route coupling. This metric describes the average number of nodes of a path that are blocked for receiving data while a node on another path transmits its data. Selecting paths with low correlation factor and route coupling offers better performances when several paths are used simultaneously as it was proved in [11] and [12]. Nevertheless, both these approaches require the computation of the network connectivity graph to select minimized interference paths, the use of such technique in dense WSNs requires therefore intensive additional calculations. In contrast to the not suitable techniques for WSNs cited above, other approaches have been used to reduce the effects of inter-path interference.

Authors in [13] proposed an interference-minimized multipath routing (I2MR) protocol that increases throughput by discovering zone-disjoint paths and distributes network traffic over the discovered paths. Based on the first shortest path discovered, I2MR marks one and two-hop neighboring nodes of all the intermediate nodes along the first path as the interference zone of the primary path and constructs interference minimized secondary and backup paths through the nodes that are not marked as the interference zone of the primary path. In [14], a Maximally Radio-Disjoint Multipath Routing (MR2) protocol is presented. MR2 addresses the interference problem between adjacent paths to satisfy the bandwidth requirements of multimedia applications. It utilizes an adaptive incremental technique to construct minimum-interfering paths. When the first path is built and becomes active, all the intermediate nodes along this active path should notify their neighboring nodes to act as the passive nodes in order to prevent them from participating in any route discovery process.

The broadcast nature of the wireless medium has been used by various multipath routing to achieve different purposes. For instance, the work in [15] proposes an adaptive iterative approach to construct minimum-interfering paths based on the broadcast nature of the wireless medium approach. A cost function, which considers, amongst other, the interference level that a node has experienced, is utilized to build such paths.

Unlike our work, none of the previously proposed solutions consider a low data rate ZigBee networks based on IEEE 802.15.4 standard and a real video traffic to evaluate the performance of the proposed multipath routing. Furthermore, the approach that blocks the neighboring nodes for constructing non-interfering paths introduces, either a highly complicated algorithm or a flooding strategy which imposes a high control overhead. In our proposed protocol, the overhearing characteristic of the wireless medium is used to construct node disjoint paths with minimum interference.

This paper is the extension of the authors previous publication [?]. We have designed and implemented a node disjoint multipath routing for ZigBee networks. The main goal was to increase the global throughput, basically insufficient in ZigBee-based WSNs, to satisfy bandwidth requirements for high traffic applications. We have showed that the quality of transmissions may be degraded due to interference, even though the paths are physically separated (node disjoint paths) and therefore, the selection of non-interfering paths is the main criterion to be addressed when simultaneous transmission through multiple paths is used.

In this article, we propose an enhanced ZigBee multipath routing taking interference into account when using simultaneous transmission data through multiple paths over ZigBee networks. The new contributions of this paper include : (i) defining a novel metric to estimate the interference level of each path during discovery phase to select multiple paths with a reduced effect of interferences, (ii) a video quality evaluation through the proposed interference-minimized ZigBee multipath routing from several Quality of Service (QoS) metrics at both the network and the application levels.

The rest of the paper is organized as follows. Section II describes two standards IEEE 802.15.4/ZigBee and MPEG-4 video, which are used in the paper. Our proposed interferenceminimized ZigBee multipath routing protocol is described in Section III and Section IV provides our simulation results for video transmission over ZigBee networks. Finally, Section V concludes the paper.

\section{IEEE 802.15.4/ZIGBEE AND MPEG-4 VIDEO}

\section{A. MAC IEEE 802.15.4 and ZigBee}

ZigBee is a robust wireless communication standard managed by the ZigBee Alliance [1] and based on the IEEE 802.15.4 physical and MAC layers standard [2]. It defines a network layer, application framework as well as security services. The MAC layer defines two types of nodes: Reduced Function Devices (RFDs) and Fully Functional Devices (FFDs) and two operational modes, non-beacon-enabled mode and beacon-enabled mode. The ZigBee network layer defines how a network address is assigned to each participant ZigBee device and how a data packet is routed. ZigBee specification extends the basic star topology of IEEE 802.15.4 to a mesh or a cluster-tree topology. Two routing protocols are considered in 
ZigBee depending on the adopted topology. The table-driven routing (similar to AODV : Ad hoc On demand Distance Vector) is used in the mesh topology while tree routing is adopted for a tree topology.

\section{B. MPEG-4 video}

MPEG-4 [?] is among the most efficient compression formats in terms of bandwidth utilization. It is one of video streaming encoding technologies which generate low bit rate video streams, therefore it is suitable for bandwidth limited wireless networks. There are three types of MPEG-4 encoded frames which are $I, P$ and $B$ frame. An $I$ frame is a frame compressed solely on the information contained in the frame, no other reference is made to any other video frames before or after it. A $P$ frame is a frame that has been compressed using the data encoded in the frame itself and the predictions from preceding $I$ or $P$ frames. $P$ frames require less bandwidth than $I$ frames. Bidirectionally $(B)$ frames are encoded using predictions from the preceding and succeeding $I$ or $P$ frames. All these different frame types are then combined in a specific repeating order to create what is known as a Group of Pictures $(G o P)$.

\section{INTERFERENCE-AWARE ZIGBEE MULTIPATH ROUTING PROTOCOL}

\section{A. ZigBee multipath routing protocol overview}

As mentionned above, the proposed multipath routing protocol is an enhanced of the protocol designed and implemented in our previous work [?]. It is a hybrid (proactive/reactive) node disjoint multipath routing protocol for ZigBee cluster tree based WSNs. To build multiple node disjoint paths, three main mechanisms were considered : (i) the MAC parent-child relationships between IEEE 802.15.4 devices in the cluster tree topology, (ii) the neighbor links maintained by every node using the neighbors table and (iii) the labelling scheme deduced from the ZigBee addressing scheme in order to apply our disjoint multipath routing. When the cluster-tree topology is formed, each node of the tree structure is assigned a label which describes the hierarchical path from the root (the PAN coordinator which corresponds to the network Sink) to it. To allow the construction of additional paths, we have, on the one hand, added new fields to the neighbors table. On the other hand, we have extended the parent-child relationships by non-tree neighbors relationships. When the node disjoint paths are built, the source node used two paths simultaneously for transmitting its data packets to the Sink. However, the data transmission quality may be degraded due to inter-path interference even if the paths are physically separated.

To improve the ZigBee multipath routing performances and make it more susceptible to support multimedia applications, we introduce a novel metric called Interference Level that calculates the interference level of the path $P$ noted $I L(P)$ based on the overhearing approach. $I L(P)$ allows selecting multiple paths by reducing the effect of inter-path interference between paths as far as possible. The discovery of completely radio disjoint paths (no nodes in the interference range of each other) is not always feasible in real wireless networks. However, selecting node disjoint paths with minimum interference allows avoiding the performance degradation of wireless network and reaching the load balancing or bandwidth aggregation goals. The calculation of $I L(P)$ is performed during the discovery phase of node disjoint paths. The same path discovery messages sent by the source and the Sink in unicast mode were used to perform this calculation. The main advantage of this solution is to not overload the network with additional control messages.

\section{B. Path interference level metric}

The basic idea to calculate the path interference level is as follow: when the source detects an event and in order to reduce the end-to-end latency, it starts immediately transmitting data packets $D P$ to the Sink using the proactive tree routing already established during the association phase at the MAC layer. Also, it starts the route discovery phase, which represents the reactive part of the multipath routing protocol, by sending the Explore message ExploreMsg to the Sink. Explore $M s g$ is forwarded (unicast and never broadcast) from node to node using forwarding decisions [?]. A node can compute the interfering nodes list when it overhears all the discovery messages from its neighbors correctly and without any loss. In instance, node 2 in Fig. 1 may know the interfering neighbors nodes belonging to other paths, namely, nodes 10 , 12 and 13 . However, node 2 may not overhear the discovery messages sent by these nodes due to link unreliability, if a message is lost, the list is incomplete. So to strengthen the interference level calculation process, the acknowledgment mechanism with ACK can be used at each hop. The data packets $D P$ transmitted to the Sink can also be used for the $I L(P)$ calculation. When the Sink receives a $D P$ or a Explore $M s g$, it replies by transmitting a $\operatorname{RepDPMsg}$ or a ReplyMsg respectively along the proactive or the reactive reverse path estabilished during the path discovery phase. Whenever the reply messages move from the Sink to the source node on the reverse path, the total number of interfering nodes with nodes belonging to path $P$ is calculated.

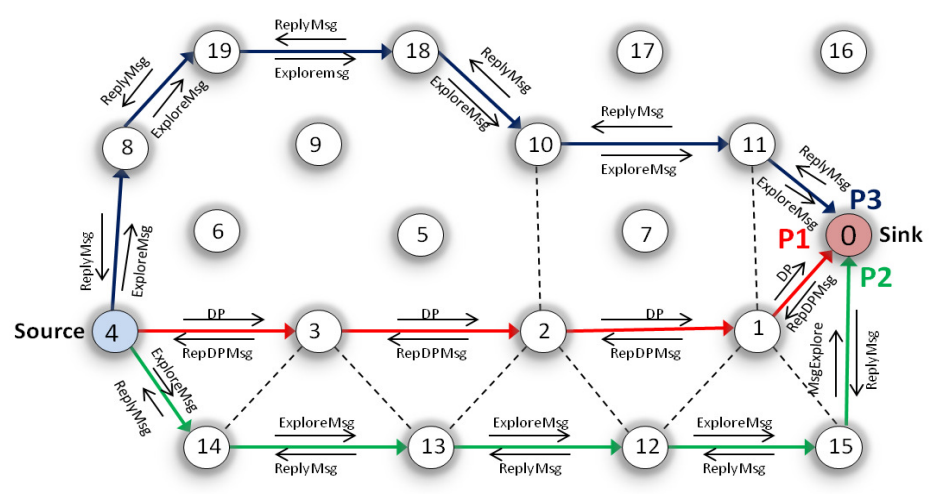

Figure 1. Path interference level computation

To calculate the interference level of a path $\mathrm{P}(I L(P))$ 
with respect to other paths, we use hereinafter, the following notations :

- $\operatorname{Long}(P)$ is the number of hops (links) of the path $P$.

- $N b(P)$ is the intermediate nodes number of the path $P$. $N b(P)=\operatorname{Long}(P)-1$.

- $I N L(i)$ represents the list of nodes belonging to other used paths that interfere with node $i$.

- INT $(P)$ represents the total nodes that cause interference with the path $P$.

Algorithm 1 is executed when node $w$ listens passively the unicast transmission of Explore $M s g$ or DP packet from the sender node $u$ to the receiver node $v$. In this situation, node $w$ is not the intended recipient of Explore $M s g$ or $D P$ packet, but it has just heard it. $I N$ (Interferent Node) is a field in the neighbors table of each network node. Initially, its value is zero, and it is sets to 1 for a neighbor when it is the source of a packet of type DP or ExploreMsg.

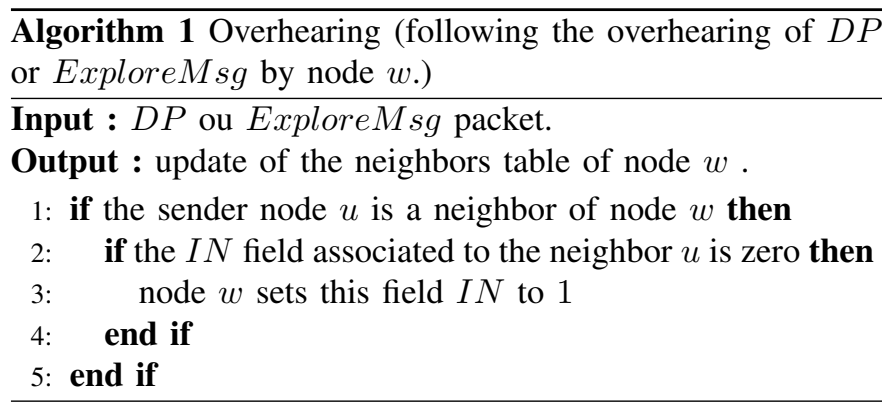

This algorithm measures the rate of overhearing that node $w$ can record from different transmissions of Explore $M s g$ or $D P$ packets carried in its vicinity. This overhearing rate is represented by the size of the list containing all neighbors of node $w$ which belong to other concurrent paths and interfere with it. When the Sink receives ExploreM sg or DP packets, it replies by transmitting ReplyMsg or RepDPMsg packets respectively along the reverse paths. When an intermediate node $u$ of the reverse path $P$ receives ReplyMsg or $R e p D P M s g$ packet, it executes algorithm 2. For the other nodes in the neighborhood who listen passively ReplyMsg or RepDPMsg packet and do not participate in routing set their $I N$ field to zero in order to discover other possible paths. $I N T$ is a field of both ReplyMsg and RepDPMsg packets. It contains the total number of nodes that interfere with nodes belonging to path $P$. When the Sink sends these packets, the initial value of $I N T$ is zero and, at each hop, this value is incremented by the number of nodes that interfere with each node along the reverse path.

Details of the three discovered node disjoint paths $P 1: 4 \rightarrow$ $3 \rightarrow 2 \rightarrow 1 \rightarrow 0, P 2: 4 \rightarrow 14 \rightarrow 13 \rightarrow 12 \rightarrow 15 \rightarrow 0$ and $P 3: 4 \rightarrow 8 \rightarrow 19 \rightarrow 18 \rightarrow 10 \rightarrow 11 \rightarrow 0$, between the source node 4 and the Sink node 0 (Fig. 1) are shown in table I. The list of interfering nodes with the node $i(I N L(i))$ is calculated from the values of the $I N$ field of each neighbor of the node $i$. They are neighbors that have the value 1 in their $I N$ field. The total of nodes that interfere with the $N b(P)$ intermediate
Algorithm 2 Calcul_INT $(P)$ when receiving a ReplyMsg or RepDPMsg by a node $u \in P$

Input : ReplyMsg or RepDPMsg packet.

Output : update the $I N T$ field.

1: for all neighbor $v$ of node $u$ do

2: if $I N$ field of node $v$ is égal to 1 then

3: $\quad$ increment the value of INT of the ReplyMsg or RepDPMsg packet

4: $\quad$ end if

5: end for

Table I

CAlcul of $I N T(P)$ AND $I L(P)$ FOR The EXAMPle of THE Fig. 1.

\begin{tabular}{|c|c|c|c|c|c|}
\hline Path & Node $i$ & INL(i) & Size of INL(i) & $\mathrm{INT}(P)$ & $\operatorname{IL}(P)$ \\
\hline \multirow{3}{*}{$P 1$} & node 3 & $\{14,13\}$ & 2 & \multirow{3}{*}{8} & \multirow{3}{*}{2.66} \\
\hline & node 2 & $\{10,12,13\}$ & 3 & & \\
\hline & node 1 & $\{12,11,15\}$ & 3 & & \\
\hline \multirow{4}{*}{$P 2$} & node 14 & $\{3\}$ & 1 & \multirow{4}{*}{6} & \multirow{4}{*}{1.5} \\
\hline & node 13 & $\{2,3\}$ & 2 & & \\
\hline & node 12 & $\{1,2\}$ & 2 & & \\
\hline & node 15 & $\{1\}$ & 1 & & \\
\hline \multirow{5}{*}{$P 3$} & node 8 & \{\} & 0 & \multirow{5}{*}{2} & \multirow{5}{*}{0.4} \\
\hline & node 19 & \{\} & 0 & & \\
\hline & node 18 & \{\} & 0 & & \\
\hline & node 10 & $\{2\}$ & 1 & & \\
\hline & node 11 & $\{1\}$ & 1 & & \\
\hline
\end{tabular}

nodes of the path $P(I N T(P))$ is calculated as follows :

$$
\operatorname{INT}(P)=\sum_{i=1}^{N b(P)} \operatorname{Size}(\operatorname{INL}(i))
$$

When the source node receives the reply packet from the discovered path $P$, it calculates the interference level of this path $I L(P)$ as a function of the total interfering nodes that interfere with this path and the number of the intermediate nodes of this path given by equation 2 .

$$
I L(P)=\frac{I N T(P)}{N b(P)}
$$

The best path is the one with the lowest value of the interference level. Table I shows that $P 3$ is the best path, following by the path $P 2$ and the last one is the path $P 1$ which is between the two other paths $P 2$ and $P 3$. In Fig. 1, we see that $P 2$ and $P 3$ are completely radio disjoint paths (spaced from each other by more than two hops), $P 1$ and $P 3$ are partielly radio disjoint paths and $P 1$ and $P 2$ are not radio disjoint paths.

Once the source node has calculated the interference level of each path, it sorts the values in ascending order and select the best pair of paths that minimize the inter-path interference for data transmission.

\section{Simulation Results}

Different simulations have been carried out to evaluate the performances of our interference-aware ZigBee multipath routing protocol with respect to the interfering ZigBee multipath routing version (without $I L(P)$ metric) and to the 
classical single ZigBee tree routing referred to Multipath-PIL, Multipath and Monopath respectively in our simulations.

For the tests, we selected one video sequence of QCIF resolution (128*128), called HallMonitor, encoded in MPEG4 format with an open source $f$ fmpeg encoder/decoder [?] and comprises 300 frames. The number of nodes in the network topology is fixed to 101 stationary nodes. Only a video sensor node captures, encodes and sends, over two paths, the video sequence to the Sink at the center of the area. The communication range is of $11 \mathrm{~m}$, it allows to cover at least three neighbors and at most eight neighbors. The nonbeacon mode with unslotted CSMA/CA mechanism of the IEEE 802.15.4 standard and the two-ray ground propagation model are used at the MAC and physical levels respectively. The MTU (Maximum Transfer Unit) size is 100 bytes. The frame rates is varied from $3 \mathrm{fps}$ to $24 \mathrm{fps}$ to evaluate different metrics for different routing strategies. All the experiments in this paper were conducted with an open source framework Evalvid [?] integrated to the simulator network NS2 with the IEEE 802.15.4 implementation provided by Zheng [16]. In our simulation study, $B$ frames are not considered in order to minimize complexity process and latency caused by this type of frames.

Video quality was evaluated from a network level point of view by using QoS metrics, such as frame loss and throughput. At the application level, an objective metric was also used to analyse the perceived video quality by calculating the frame Peak-Signal-to-Roise Ratio $(P S N R)$ [?].

The results show that when the number of frames per second increases from $3 \mathrm{fps}$ to $24 \mathrm{fps}$, the network performance degrades due to the high rate of video frames loss which negatively affects the video quality received at the Sink. This is true for all the routing strategies. However, for a video traffic load less than or equal to $6 \mathrm{fps}$ (not exceeding the theoretical capacity of the network bandwidth $250 \mathrm{kbps}$ ), we note that the non-interfering multi-path routing MultipathPIL is more efficient than the two other routing strategies Monopath and Multipath in terms of frame loss rates (Fig. 2, Fig.3), throughput (Fig. 4) and video quality measured by PSNR (Fig. 5). These results are explained by the fact that, when the network is overloaded, the single-path routing Monopath can not handle such a traffic load that exceeds its capacity while the inter-path interferences increase in the case of Multipath routing strategy, which lead to high frame loss rates that deteriorates the video quality.

MPEG-4 has particular characteristics. The received video quality not only depends on the frames loss rate but also on the nature of the lost data. Indeed, for MPEG-4 video, $I$ frames provide reference information and are necessary for decoding $P$ frames and therefore the loss of $I$ frames has a greater impact on the quality of the received video than the loss of a frame of type $P$. As we can see in Fig. 3, the use of Multipath$P I L$ routing strategie reduces the percentage of $I$ frames loss which ensures video playback improved for the user. These simulation results are consistent with those in Fig. 5, this can be explained by the fact that the lost of frames containing the most important information leads to a severe degradation of video quality.

The results also show that Monopath and Multipath routing strategies have almost the same behavior despite that the multipath routing approach offers several benefits over the monopath routing technique. These findings confirms that the use of simultaneous data transmission over multiple paths can be effective unless the effects of inter-path interference are minimized.

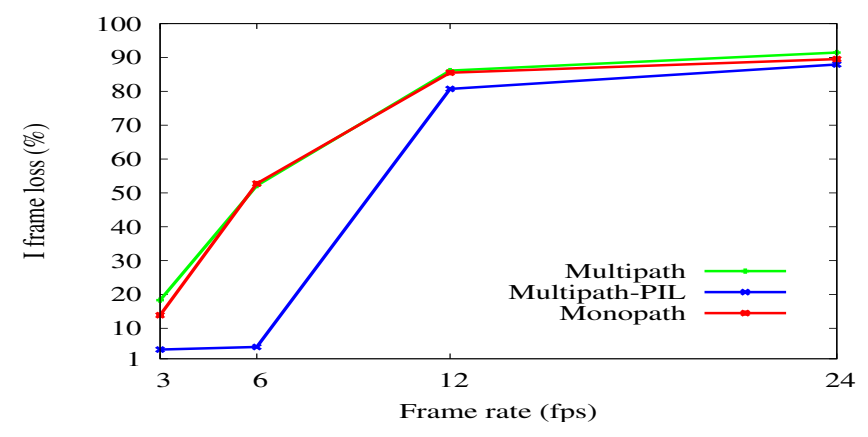

Figure 2. I Frame loss rate for different frame rates

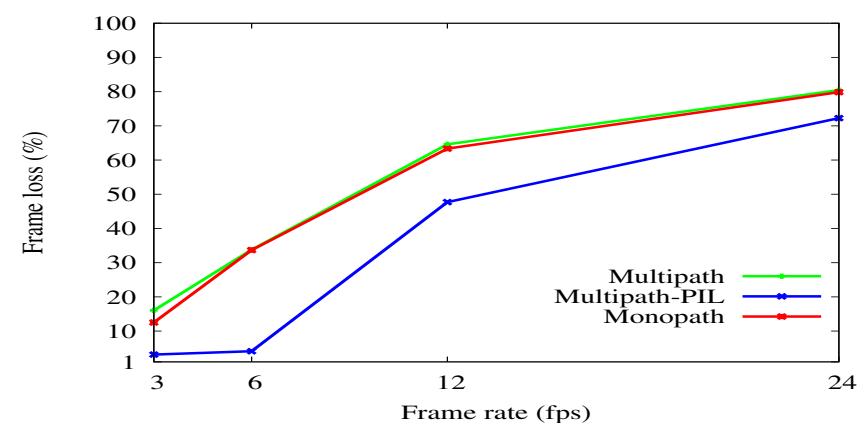

Figure 3. Frame loss rate for different frame rates

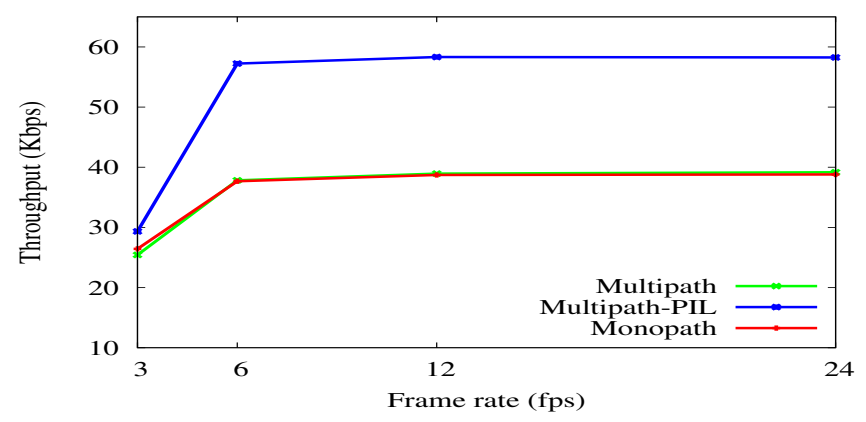

Figure 4. Throughput for different frame rates

Fig. 6 displays a frame selected randomly from the transmitted video. It shows the benefits of transmitting simultaneously video traffic using the proposed protocol by comparing the received frame (Fig. 6(b)) with the other solutions (Fig. 6(c), Fig. 6(d)) and also with the original frame (Fig. 6(a)). The 


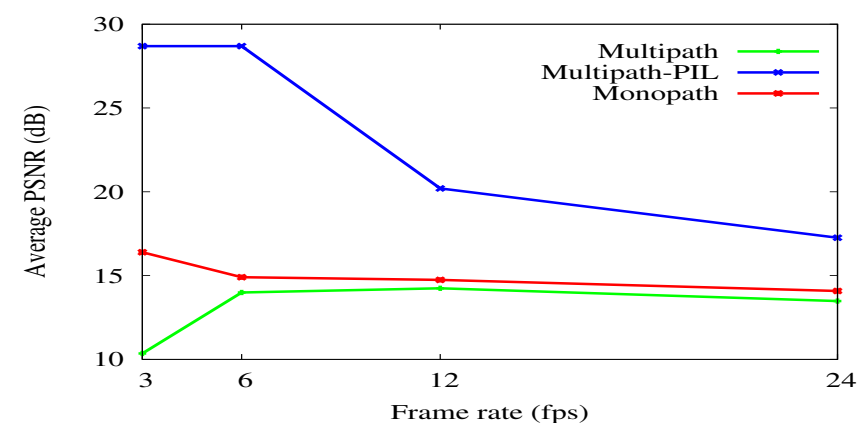

Figure 5. PSNR for different frame rates

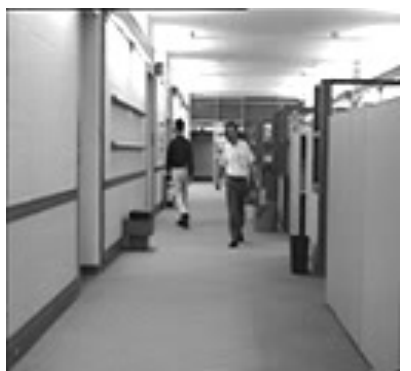

(a) Original

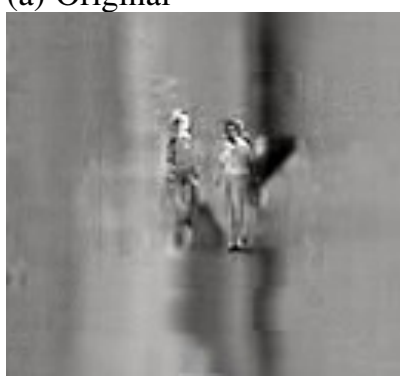

(c) Video-aware Monopath

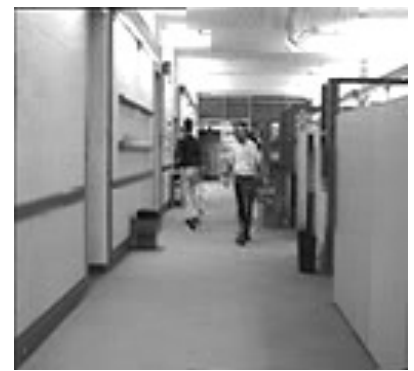

(b) Video-aware Multipath-PIL

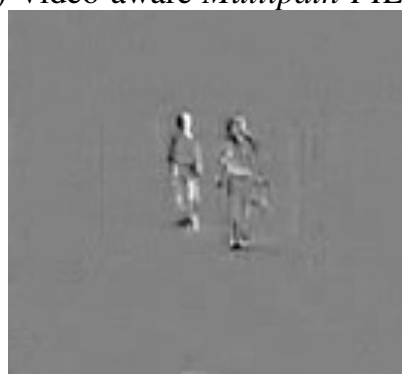

(d) Video-aware Multipath

Figure 6. Frame 216 of Hall Monitor sequence video

frames transmitted via Monopath and Multipath present a higher video distortion compared to the original frame and the frame transmitted via interference-minimized multipath routing protocol.

\section{CONCLUSion}

In this paper, we presented a multipath routing for ZigBee based WSNs to handle multimedia applications taking into account the effects of inter-path interference. The multipath routing tries to establish multiple node disjoint paths from the source to the Sink, calculates the interference level for each discovered path and gets to select the most noninterfering paths to send video traffic. Simulation results show that the proposed interference-minimized ZigBee multipath routing provides the best performances with regard to the two other strategies routing. This was found by evaluating the proposed protocol through QoS metrics and the well known objective metric $P S N R$ as well as showing video frames. Thus, we can conclude that the proposed protocol is a multipath solution which provides a good human acceptable

video quality required for applications like video surveillance where high video quality is not needed.

\section{REFERENCES}

[1] “Zigbee alliance. zigbee document 02130r9: Network specification,” July 2004.

[2] "Ieee standards part 15.4: Wireless medium access control (mac) and physical layer (phy) specifications for low-rate wireless personal area networks (lr-wpans)," New York, October 2003.

[3] I. E. G. Richardson, H.264 and MPEG-4 Video Compression Video Coding for Next-generation Multimedia. John Wiley and Sons Ltd, 2003.

[4] F.-S. A.J.Garcia-Sanchez, J.Garcia-Haro, and F.Losilla, "A cross-layer solution for enabling real-time video transmission over IEEE 802.15.4 networks, published online," Multimedia Tools and Applications, February 2010.

[5] F.QIN, "Technologies to improve the performance of wireless sensor networks in high-traffic applications," Ph.D. dissertation, Department of Electronic and Electrical Engineering University College London, 2012.

[6] W. Zijian, E. Bulut, and B. K. Szymanski, "Energy efficient collision aware multipath routing for wireless sensor networks," in Proceedings of the 2009 IEEE International Conference on Communications (ICC'09), Dresden, Germany, 2009, pp. 91-95.

[7] S. Roy, S. Bandyopadhyay, T. Ueda, and al, "Multipath routing in ad hoc wireless networks with omni directional and directional antenna: A comparative study," in Proceedings of the 4th International Workshop on Distributed Computing, Mobile and Wireless Computing (IWDC'02), Calcutta, India, 2002, pp. 184-191.

[8] W. H. Tarn and Y. C. Tseng, "Joint multi-channel link layer and multipath routing design for wireless mesh networks," in Proceedings of the 26th IEEE International Conference on Computer Communications (INFOCOM'07), Anchorage, AK, USA, 2007, pp. 2081-2089.

[9] R. Nelson and L. Kleinrock, "Spatial TDMA: A collision-free multihop channel access control," IEEE Transactions on Communications, vol. 33 , no. 9, pp. 934-944, September 1985.

[10] W. Wei and A. Zakhor, "Interference aware multipath selection for video streaming in wireless ad hoc networks," IEEE Transactions on Circuits and Systems for Video Technology, vol. 19, no. 2, pp. 165-178, 2009.

[11] K. Wu and J. Harms, "Performance study of a multipath routing method for wireless mobile ad hoc networks," in Proceedings of the 9th International Symposium on Modeling, Analysis and Simulation of Computer and Telecommunication Systems, Cincinnati, OH, USA, August 2001, pp. 99-107.

[12] M. Pearlman, Z. Haas, P. Sholander, and S. Tabrizi, "On the impact of alternate path routing for load balancing in mobile ad hoc networks," in Proceedings of the 1st ACM International Symposium on Mobile Ad Hoc Networking and Computing, IEEE Press, Piscataway, NJ, USA, 2000, pp. 3-10.

[13] J. Y. Teo, Y. Ha, and C. K. Tham, "Interference-minimized multipath routing with congestion control in wireless sensor network for high-rate streaming." IEEE Transactions on Mobile Computing Mobile Computing, , vol. 7, no. 9, pp. 1124-1137, 2008.

[14] M. Maimour, "Maximally radio-disjoint multipath routing for wireless multimedia sensor networks," in Proceedings of the 4th ACM workshop on Wireless multimedia networking and performance modeling colocated with MSWIM'08, Vancouver, Canada, 27-31 October 2008.

[15] M. Radi, B. Dezfouli, K. Bakar, S. A. Razak, and M. Nematbakhsh, "Interference-aware multipath routing protocol for qos improvement in event-driven wireless sensor networks," Tsinghua Sci. Tech, vol. 16, no. 5, pp. 475-490, 2011.

[16] M. Zheng, "NS2 simulator for IEEE 802.15.4," 2004, http://ees2cy.engr. ccny.cuny.edu/zheng/pub/. 